\title{
Special Issue "Methods and Applications of Virtual and Augmented Reality in Geo-Information Sciences"
}

\author{
Thomas P. Kersten ${ }^{1}\left[\right.$ [ $\cdot$ Dennis Edler ${ }^{2}$ (1)
}

Published online: 17 March 2020

(c) Deutsche Gesellschaft für Photogrammetrie, Fernerkundung und Geoinformation (DGPF) e.V. 2020

Due to rapid technological development and recent advances in hardware and software technology, Virtual Reality (VR) is becoming an increasingly accessible and important tool for multiple applications in science, industry and education. VR is understood as the visualisation and simultaneous perception of reality and its physical characteristics within an interactive virtual environment, which is generated by a computer in real time. In recent years, VR has found applications in a broad range of fields, including medicine (primarily in training and therapy), architecture and cultural heritage, training simulations (e.g., fire brigade, rescue services, military), construction planning and product development, the growing gaming industry, computer vision and many others. The sense of immersion achieved through virtual 3D environments offers numerous advantages, especially for the visualisation and communication in real-time of geographical data, which has traditionally been depicted in 2D maps or pseudo-3D models on the monitor screen.

Progress in sensor technologies has also spurred the growth of a market for Augmented Reality (AR). AR is the computer-aided extension of observable reality through the combination of reality and virtuality. More commonly, however, AR is understood as the visual representation of additional information as a layer over the top of an image of reality. This could be, for example, the use of computergenerated content or virtual objects, including images, sound or videos, superimposed over the world by AR glasses or a

Thomas P. Kersten

Thomas.Kersten@hcu-hamburg.de

Dennis Edler

Dennis.Edler@ruhr-uni-bochum.de

1 Labor für Photogrammetrie \& Laserscanning, HafenCity Universität Hamburg, Überseeallee 16, 20457 Hamburg, Germany

2 Ruhr-Universität Bochum, Geographisches Institut, AG Geomatik, Universitätsstr. 150, 44801 Bochum, Germany smartphone. The decisive criterion is the superimposition of virtual content over reality in real time, with a continuous adaptation to the point of view of the user.

The technical capabilities in the fields of VR and AR have become increasingly obvious in our everyday lives. It is, therefore, no surprise that the development of VR/AR applications with compatible spatial data and data formats is a new challenge for the community of Geo-information Science, incl. Photogrammetry, Remote Sensing/Earth Observation, Spatial Cognition, Citizen Science, Geography and Education. This special issue includes a selection of papers that gives an overview of the state-of-the-art research and applications in VR and AR. Five original papers and two technical reports incorporate both experimental investigations and applied research.

Nagy has developed a new method for improving the azimuth determination by skyline extraction to identify mountains in an AR application. Büyüksalih et al. present a workflow from 3D laser scanning to Virtual Reality visualisation for the Istanbul Çatalca İnceğiz Caves. The VR model serves as a basis for preserving knowledge of the past through virtual visits of the cultural heritage monument. Schmohl et al. introduce the "Stuttgart City Walk" case study, allowing the general public to visualise textured meshes of digital surface models using Virtual Reality. They were able to draw upon CityGML data sets and textured 3D models provided by the open library 3D warehouse. Hruby et al. address the topic of spatial presence and immersion in geo-environments. Based on WorldView-2 data, bathymetric data and habitat maps, they created an immersive underwater environment. Statistical evidence is provided that immersive technologies significantly influence the user's sense of spatial presence in VR environments. Another experimental study was conducted by Keil et al. The team investigated the technical capabilities of Microsoft HoloLens for Augmented Reality in indoor navigation. Their study is focused on the impact of artificial signifiers (a continuous grid system) in a $3 \mathrm{D}$ indoor environment on spatial cognition performance. 
The empirical data point to a significant advantage of AR for distance estimation tasks, whereas AR did not show an advantage for the performance in spatial memory.

Two technical reports present practical applications of AR and VR. Stylianidis et al. demonstrate how Augmented Reality could be used for the visualisation of underground utilities, while Rienow et al. use satellite-borne and ISSborne remote sensing data for Augmented Reality and Virtual Reality applications to support knowledge transfer and e-learning in school lessons.

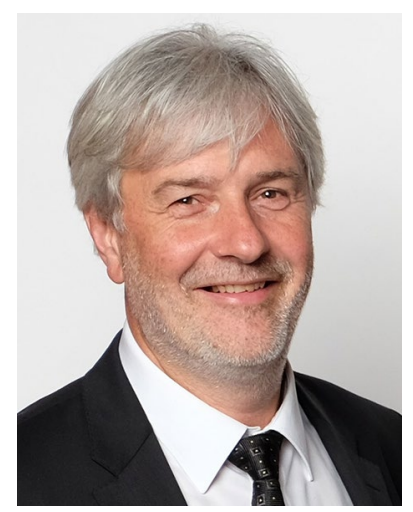

Guest Editor.

Thomas P. Kersten (Photogrammetry \& Laser Scanning Lab, HafenCity University Hamburg).
This special issue is organised by the new joint commission "Virtual and Augmented Reality" of DGPF and DGfK (German Cartographic Society). It was founded in March 2019. The aim of this commission is to promote VR/AR within the community of spatial sciences and neighbouring disciplines, to establish a network of scientists and users interested in VR/ AR, to organise workshops about this topic and sessions at the annual meetings of both societies. Interested researchers and users are invited to join the new commission VR/AR for active participation (https://www.dgpf.de/aks/var.html).

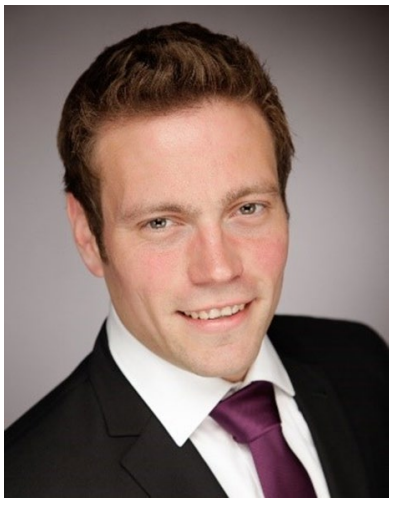

Guest Editor.

Dennis Edler (Institute of Geography, Ruhr-University Bochum). 